\title{
A new laryngeal mask supraglottic airway device with integrated balloon line: a descriptive and comparative bench study
}

This article was published in the following Dove Press journal:

Medical Devices: Evidence and Research

2 November 2016

Number of times this article has been viewed

\section{YingHai Zhou' \\ Korinne Jew ${ }^{2}$}

'Research \& Development, Patient Monitoring \& Recovery, Medtronic Technology Center, Shanghai, People's Republic of China; ${ }^{2}$ Medical Affairs, Minimally Invasive Therapies Group, Medtronic, Boulder, CO, USA
Correspondence: YingHai Zhou

China Technology Center, 6F, Building 3, 2388 Chenhang Road, Shanghai, 20III4,

People's Republic of China

Tel +862133230127

Fax +862133230222

Email renny.zhou@medtronic.com
Abstract: Laryngeal masks are invasive devices for airway management placed in the supraglottic position. The Shiley ${ }^{\mathrm{TM}}$ laryngeal mask (Shiley ${ }^{\mathrm{TM}} \mathrm{LM}$ ) features an integrated inflation tube and airway shaft to facilitate product insertion and reduce the chance of tube occlusion when patients bite down. This study compared the Shiley LM to two other disposable laryngeal mask devices, the $\mathrm{Ambu}^{\circledR}$ AuraStraight ${ }^{\mathrm{TM}}$ and the LMA Unique ${ }^{\mathrm{TM}}$. Overall device design, tensile strength, flexibility of various structures, and sealing performance were measured. The Shiley LM is structurally stronger and its shaft is more resistant to compression than the other devices. The Shiley LM is generally less flexible than the other devices, but this relationship varies with device size. Sealing performance of the devices was similar in a bench assay. The results of this bench study demonstrate that the new Shiley LM resembles other commercially available laryngeal mask devices, though it exhibits greater tensile strength and lower flexibility.

Keywords: laryngeal mask, supraglottic airway, supralaryngeal device

\section{Introduction}

Supralaryngeal or supraglottic airway devices are placed above the larynx to maintain an open airway during anesthesia without endotracheal penetration of the device. Defined under ISO 11712, these devices seal and isolate the supralaryngeal area providing a hands-free alternative to face masks and a less invasive alternative to tracheal devices. The first supralaryngeal device, the laryngeal mask, was introduced in 1988 and obtained US Food and Drug Administration clearance for medical use as an alternative to face masks during anesthesia in $1991 .{ }^{1}$ Structurally, supralaryngeal masks include a shaft attached to a dome surrounded by an inflatable cuff that covers the periglottic tissues. ${ }^{1}$ Since the approval of the first laryngeal mask, other similar and dissimilar supralaryngeal airway devices have been introduced, and there are now more than 30 related devices. $^{2}$ A disposable, single-use laryngeal mask, the LMA Unique ${ }^{\mathrm{TM}}$ (LMA-U), was introduced in $1997 .{ }^{3}$ Despite being stiffer than the reusable laryngeal mask, the LMA-U was found to have similar ease of insertion and similar low incidence of reported sore throat after use. ${ }^{3}$

Because laryngeal masks are quick and relatively easy to use, they have found utility in a wide range of clinical situations, including anesthesia, resuscitation, and intensive care. ${ }^{2}$ Laryngeal masks were initially used for spontaneously breathing patients, but innovations have made it possible to use the devices with positive pressure ventilation in patients not breathing on their own. ${ }^{2}$ The devices may be used as an alternative to mask anesthesia for short procedures in the operating room. Laryngeal masks are 
absolutely contraindicated in patients with limited oral opening and upper airway obstruction. Relative contraindications exist for the morbidly obese, people who have not fasted, and others with increased risk of aspiration, as well as patients with upper gastrointestinal bleeding and pregnant patients in the second and third trimesters. ${ }^{4,5}$ The increasing use of laryngeal masks in out-of-hospital settings necessitates that the devices be easy to insert correctly, even by inexperienced personnel.

The Shiley ${ }^{\mathrm{TM}}$ laryngeal mask (Shiley LM) is a disposable, single-use supraglottic airway device that introduces an integrated airway and cuff-inflation tube (Figure 1). This integration was designed to provide easier insertion and reduced opportunity for the inflation tube to become crimped or be bitten by the patient. The main objective of this study was to compare the physical characteristics of the Shiley LM to those of other disposable laryngeal masks, the Ambu ${ }^{\circledR}$ AuraStraight $^{\mathrm{TM}}$ (AA) and the LMA-U, to test the hypothesis that these devices are similar with respect to flexibility, stability, and sealing performance.

\section{Materials and methods Descriptive comparison of devices}

The curvature and inner diameter of the shaft of various sizes of each device were compared. Three-dimensional scanning (OpticScan-3M, Ricoh Imaging Company, Ltd, Tokyo, Japan) was used to measure the curvature of the shaft of various sizes of the LMA-U and AA. The inner diameters of the shafts of the LMA-U and AA were measured and compared to the product design dimension for the shaft of the Shiley LM.

\section{Shaft flexibility}

Shaft flexibility was assessed by bending the shaft into a " $U$ " shape and measuring the force, in $\mathrm{N}$ (newtons), applied by the shaft on a test probe. Measurements were made with an advanced tensile and compression testing machine (ESM301L,

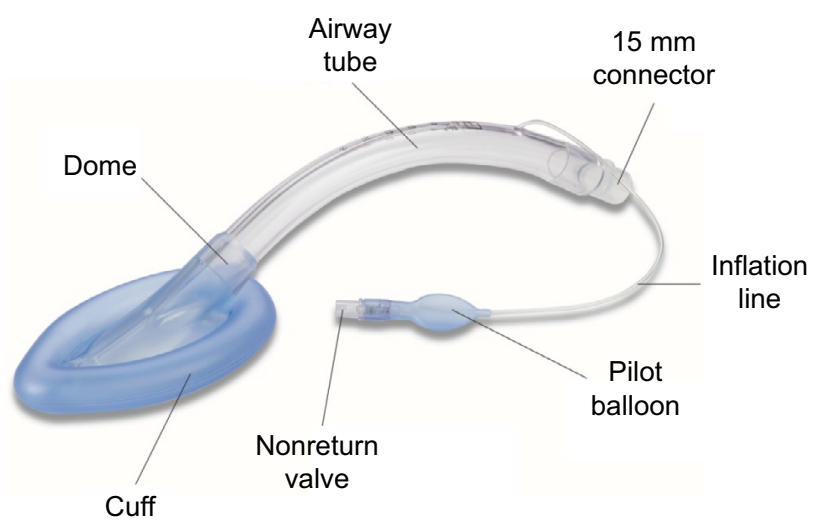

Figure I The Shiley ${ }^{\mathrm{TM}}$ laryngeal mask with integrated inflation tube and airway shaft.

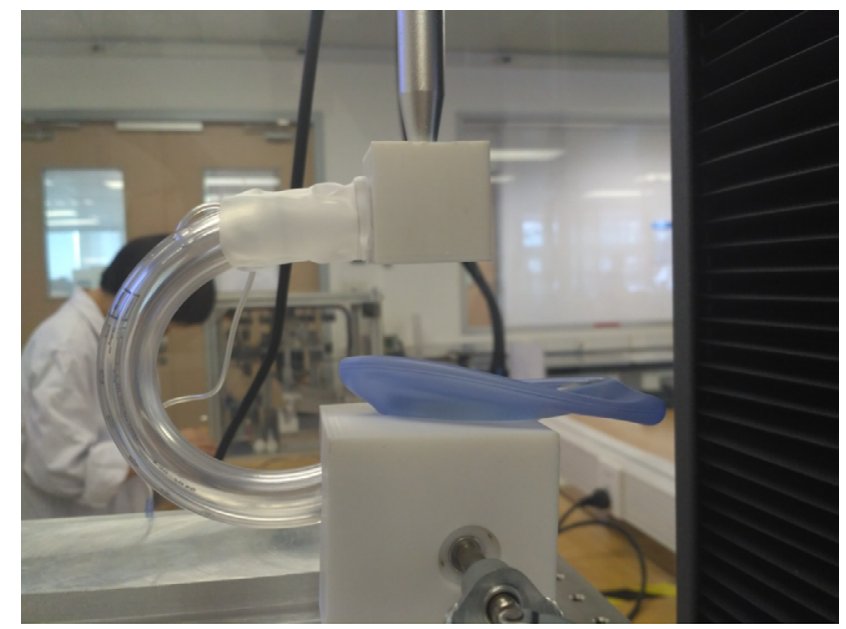

Figure 2 Measurement of shaft flexibility.

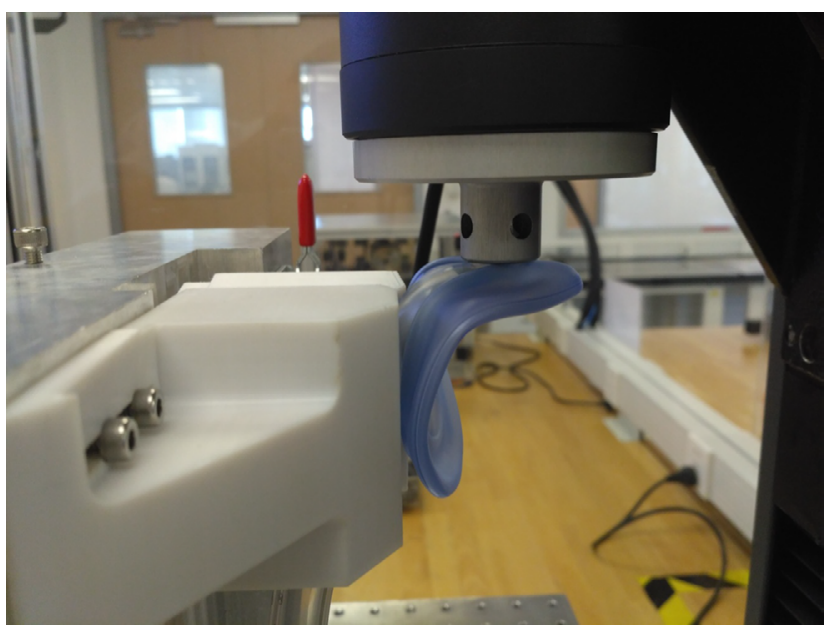

Figure 3 Measurement of dome and cuff flexibility.

Mark-10 Corporation, Copiague, NY, USA), a motorized test stand designed for tension and compression testing up to 1,500 $\mathrm{N}$ (Figure 2). The axis of the $15 \mathrm{~mm}$ connector was positioned parallel to the outside ridgeline of the dome. Each specimen was maintained in the test position for 10 seconds, and the maximum force was recorded. A minimum of ten specimens of sizes 1,3 , and 5 of each device were tested.

\section{Flexibility of dome and cuff}

The flexibility of the dome and cuff was assessed by bending the tip of the dome to form a right angle and measuring the force applied by the product on a test probe. Measurements were made using a tensile and compression testing machine (5944U2137 MicroTester, Instron, Norwood, MA, USA), an instrument designed for carrying out multiple mechanical tests, including tension, compression, and sensitive load measurement on small specimens (Figure 3). The maximum force was recorded. A minimum of ten specimens, each of sizes 1, 3, and 5 of each device, were tested. 


\section{Tensile strength}

The tensile strength of bonding at the following junctures was tested: dome to cuff, dome to airway tube, airway tube to the connector, and pilot balloon to inflation tube. Each component was tested to failure, and the maximum force was recorded with a tensile and compression testing instrument (Instron) using either a $100 \mathrm{~N}$ or $10 \mathrm{kN}$ load cell depending on the required force. The tests were carried out on size 1 specimens of the Shiley LM and the LMA-U devices. The AA device has an integrated dome and airway tube and was not tested due to this design difference.

\section{Sealing performance}

A laryngeal mask cuff, inflated to $60 \pm 2 \mathrm{~cm} \mathrm{H}_{2} \mathrm{O}$, was pressed on a flat glass surface and air was evacuated from the connector by a standard syringe pump, forming a vacuum inside the laryngeal mask. A water-based lubricant was applied to the exterior of the cuff. The vacuum pressure was controlled at $-60 \mathrm{cmH}_{2} \mathrm{O}$. The inner pressure was measured after 20 seconds. The vacuum pressure at the end of the 20 -second period was subtracted from the pressure at the start of the test, and the difference in pressure was used to determine the volume of air that had passed between the cuff and the glass surface. A minimum of ten specimens, representing sizes 1, 3 , and 5 of each device type, were tested.

\section{Shaft compressibility}

During the clinical use of a laryngeal mask, it is possible that the patient may bite the shaft of the tube. Therefore, the compression resistance of the shaft of each of the three devices was measured. Ten specimens each of sizes 1, 3, and 5 were assessed. A ventilator was connected to the device and set to a specific airflow depending on product size (Table 1). The pressure at the $15 \mathrm{~mm}$ connector was measured. A compression force was then applied to the airway tube using a probe designed to mimic teeth (Table 1), and the change in pressure at the $15 \mathrm{~mm}$ connector was measured using a pressure meter (DPM2Plus, Fluke Biomedical Corporation, Everett, WA, USA) designed to measure positive and negative pressures of medical devices in either liquid or gaseous form (Figure 4). The drop in pressure after compression was reported.

Table I Settings during measure of shaft compressibility

\begin{tabular}{lll}
\hline Product size & Airflow (L/min) & Compression force (N) \\
\hline 1 & 15 & 12 \\
3 & 60 & 40 \\
5 & 60 & 50 \\
\hline
\end{tabular}

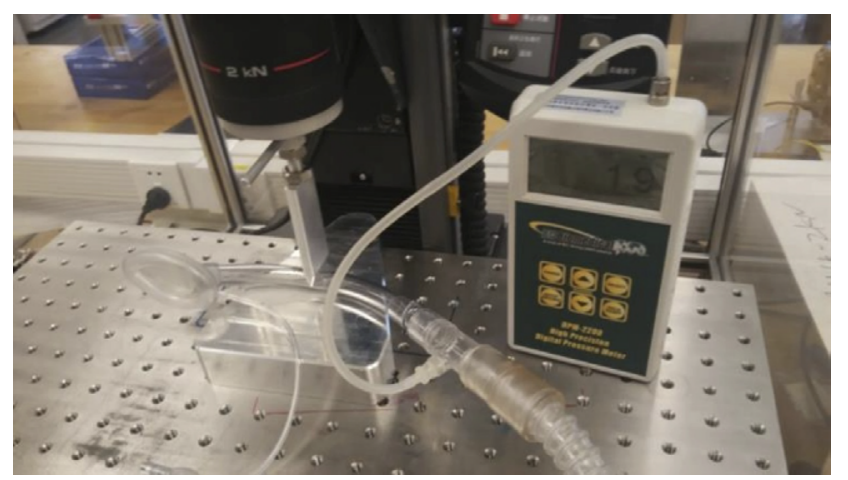

Figure 4 Measurement of shaft compressibility.

\section{Statistics}

All data were reported as mean and standard deviation. The statistical analysis of pairwise comparisons of means was conducted using two-sample $t$-tests. A difference between means was considered statistically significant when the $P$-value was $<0.05$.

\section{Results \\ Configuration}

All three devices consist of a dome surrounded by an inflatable cuff, a shaft, and an inflation system for inflation of the cuff. The Shiley LM differs from the LMA-U and the AA in that the inflation tube of the Shiley LM is integrated into the shaft (Figure 1) while in the other devices, the inflation tube is separated from the shaft with one end bonded to the cuff.

\section{Shaft curvature}

The track of LM insertion is a curve from the patient's hard palate to the laryngopharynx, passing through the soft palate. The curvature of the product shaft should approximate this curve for easiest insertion. The curvature of the shaft of various sizes of the Shiley LM, LMA-U, and AA is shown in Table 2. The minimum radius was recorded. The shaft of the Shiley LM is designed to have the same curvature as an endotracheal tube, which is a constant curvature for all device sizes. In contrast, the shafts of the LMA-U and AA vary with the device size.

\section{Minimum internal diameter of the shaft}

The internal diameter of the shaft determines airflow and thus is associated with the work of breathing. The minimum internal diameter of the shaft of various sizes of Shiley LM, LMA-U, and AA is shown in Table 2. The inner diameter of the shaft of the Shiley LM is larger than that of the others for device sizes 1 through 2.5. 
Table 2 Shaft curvature and minimum internal diameter

\begin{tabular}{|c|c|c|c|c|c|c|}
\hline \multirow[t]{2}{*}{$\overline{\text { Size }}$} & \multicolumn{3}{|l|}{ Radius (mm) } & \multicolumn{3}{|c|}{ Minimum internal diameter $(\mathrm{mm})$} \\
\hline & Shiley ${ }^{T M}$ LM & LMA-U & AA & Shiley ${ }^{\mathrm{TM}}$ LM & LMA-U & AA \\
\hline $\mathrm{I}$ & 139.7 & 73.8 & 109.31 & 5.99 & 5.16 & 5.06 \\
\hline 1.5 & 139.7 & 71.2 & Not measured ${ }^{a}$ & 6.79 & 6.43 & Not measured \\
\hline 2 & 139.7 & 87.06 & 135.34 & 8.59 & 6.73 & 7.05 \\
\hline 2.5 & 139.7 & 92.14 & Not measured ${ }^{\mathrm{a}}$ & 8.59 & 8.30 & Not measured \\
\hline 3 & 139.7 & 131.82 & 205.28 & 9.60 & 9.82 & 9.79 \\
\hline 4 & 139.7 & $|40.3|$ & 168.10 & 9.60 & 9.82 & 10.17 \\
\hline 5 & 139.7 & 142.6 & 222.44 & 11.10 & 10.92 & 11.14 \\
\hline 6 & 139.7 & No size $6^{\mathrm{b}}$ & Not measured & 11.10 & No size $6^{\mathrm{b}}$ & Not measured \\
\hline
\end{tabular}

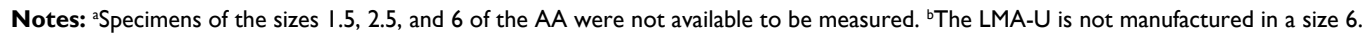

Abbreviations: AA, Ambu $^{\circledR}$ AuraStraight $^{\mathrm{TM}}$; LMA-U, LMA Unique ${ }^{\mathrm{TM}}$; Shiley ${ }^{\mathrm{TM}} \mathrm{LM}$, Shiley ${ }^{\mathrm{TM}}$ laryngeal mask.

Table 3 Shaft and dome/cuff flexibility

\begin{tabular}{|c|c|c|c|c|c|c|}
\hline \multirow[t]{3}{*}{ Size } & \multirow{2}{*}{\multicolumn{3}{|c|}{$\begin{array}{l}\text { Shaft flexibility } \\
\text { Force exerted on probe }(\mathbf{N})\end{array}$}} & \multirow{2}{*}{\multicolumn{3}{|c|}{$\begin{array}{l}\text { Dome/cuff flexibility } \\
\text { Force exerted on probe }(\mathrm{N})\end{array}$}} \\
\hline & & & & & & \\
\hline & Shiley $^{\mathrm{TM}}$ LM & LMA-U & AA & Shiley ${ }^{\mathrm{TM}}$ LM & LMA-U & AA \\
\hline \multirow[t]{2}{*}{ I } & $2.06(0.14)$ & $0.93(0.10)$ & $1.23(0.10)$ & $1.85(0.08)$ & $0.82(0.04)$ & $0.98(0.06)$ \\
\hline & & $P<0.000 I^{*}$ & $P<0.000 I^{*}$ & & $P<0.000 I^{*}$ & $P<0.000 I^{*}$ \\
\hline \multirow[t]{2}{*}{3} & $2.70(0.10)$ & $2.00(0.13)$ & $2.66(0.13)$ & $1.98(0.18)$ & $1.26(0.12)$ & I.8I (0.09) \\
\hline & & $P<0.000 I^{*}$ & $P=0.2053^{*}$ & & $P<0.000 I^{*}$ & $P<0.000 I^{*}$ \\
\hline \multirow[t]{2}{*}{5} & $2.68(0.13)$ & $2.60(0.16)$ & $3.05(0.09)$ & $\mathrm{I} .84(0.07)$ & $1.50(0.09)$ & $2.60(0.09)$ \\
\hline & & $P=0.03 \mid 8^{*}$ & $P<0.000 I^{*}$ & & $P<0.000 I^{*}$ & $P<0.000 I^{*}$ \\
\hline
\end{tabular}

Notes: Data are presented as mean (standard deviation). *Significance compared to Shiley TM LM.

Abbreviations: AA, Ambu ${ }^{\circledR}$ AuraStraight $^{\mathrm{TM}}$; LMA-U, LMA Unique ${ }^{\mathrm{TM}}$; Shiley ${ }^{\mathrm{TM}}$ LM, Shiley ${ }^{\mathrm{TM}}$ laryngeal mask.

\section{Shaft flexibility}

The flexibility of the LM shaft affects the ease of insertion; a more flexible shaft may provide better compliance with patient anatomy but may make it more difficult to convey required force during insertion. The flexibility of the shaft of three sizes of the Shiley LM, LMA-U, and AA is shown in Table 3. The shafts of all three sizes of the Shiley LM were less flexible than those of the corresponding sizes of the LMA-U $(P<0.05)$, and the shafts of the sizes 1 and 5 were less flexible than those of the corresponding sizes of the AA $(P<0.05)$.

\section{Dome and cuff flexibility}

Flexibility of the LM dome and cuff affects LM insertion and function; increased flexibility may be associated with formation of an effective seal and prevention of air leaks, but may also be associated with increased opportunity for folding during insertion. The flexibility of the dome and cuff of three sizes of the Shiley LM, LMA-U, and AA is shown in Table 3. The dome and cuff of all three sizes of the Shiley LM were less flexible than those of the corresponding sizes of the LMA-U $(P<0.05)$. The dome and cuff of the sizes 1 and 3 of the Shiley LM were less flexible than those of the corresponding sizes of the AA, while the dome and cuff of the size 5 of the Shiley LM was more flexible than those of the AA size $5(P<0.05)$.
Table 4 Tensile strength

\begin{tabular}{llll}
\hline Connection tested & \multicolumn{2}{l}{ Maximum force before failure (N) } & P-value \\
\cline { 2 - 3 } & Shiley ${ }^{\text {TM }}$ LM & LMA-U & \\
\hline Cuff-dome & 97.69 & 32.54 & $<0.0001$ \\
Dome-tube & 152.04 & 127.68 & 0.0142 \\
Connector-tube & 338.33 & 289.74 & 0.0018 \\
Inflation tube-pilot & 35.03 & 29.01 & 0.0004 \\
\hline
\end{tabular}

Abbreviations: LMA-U, LMA Unique ${ }^{\mathrm{TM}}$; Shiley ${ }^{\mathrm{TM}}$ LM, Shiley ${ }^{\mathrm{TM}}$ laryngeal mask.

\section{Tensile strength}

The force required to cause failure of various connections in the size 1 of Shiley LM and LMA-U is shown in Table 4. In every case, the bonding of components of the Shiley LM was stronger than that of the components of the LMA-U. The AA device has a different design, with an integrated dome and airway tube, so it was not tested.

\section{Sealing performance}

The sealing performance of three sizes of the Shiley LM, LMA-U, and AA was assessed by measuring the volume of air that leaked from the device after creating a vacuum between the cuff and dome and a flat glass surface. The results are shown in Table 5. The sizes 1 and 5 of the Shiley LM performed similarly to the corresponding sizes of the 
Table 5 Sealing performance

\begin{tabular}{|c|c|c|c|c|c|c|}
\hline \multirow[t]{2}{*}{ Size } & \multicolumn{3}{|c|}{ Air leak $\left(\mathrm{cmH}_{2} \mathrm{O}\right)$} & \multicolumn{3}{|c|}{ Pressure difference after compression of shaft $\left(\mathrm{cmH}_{2} \mathrm{O}\right)$} \\
\hline & Shiley ${ }^{\mathrm{TM}}$ LM & LMA-U & AA & Shiley ${ }^{\mathrm{TM}} \mathbf{L M}$ & LMA-U & AA \\
\hline \multirow[t]{2}{*}{$\mathrm{I}$} & $1.32(0.918)$ & $9.43(1.880)$ & $1.30(1.418)$ & $0.27(0.157)$ & $9.43(1.880)$ & $4.92(0.689)$ \\
\hline & & $P<0.000 I^{*}$ & $P=0.9630^{*}$ & & $P<0.000 I^{*}$ & $P<0.000 I^{*}$ \\
\hline \multirow[t]{2}{*}{3} & $0.25(0.439)$ & $19.66(2.383)$ & $\mathrm{I} .00(0.47 \mathrm{I})$ & $3.63(0.347)$ & $19.66(2.383)$ & $7.35(1.455)$ \\
\hline & & $P<0.000 I^{*}$ & $P<0.000 I^{*}$ & & $P<0.000$ I* & $P<0.000 I^{*}$ \\
\hline \multirow[t]{2}{*}{5} & $1.30(0.949)$ & $20.26(5.032)$ & $1.60(0.966)$ & $1.38(0.249)$ & $20.26(5.032)$ & $4.90(0.769)$ \\
\hline & & $P<0.000 I^{*}$ & $P=0.4925^{*}$ & & $P<0.000 I^{*}$ & $P=0.7278^{*}$ \\
\hline
\end{tabular}

Notes: Data are presented as mean (standard deviation). *Significance compared to Shiley ${ }^{\text {TM }}$ LM.

Abbreviations: AA, Ambu ${ }^{\circledR}$ AuraStraight ${ }^{\mathrm{TM}}$; LMA-U, LMA Unique ${ }^{\mathrm{TM}}$; Shiley ${ }^{\mathrm{TM}}$ LM, Shiley ${ }^{\mathrm{TM}}$ laryngeal mask.

$\mathrm{AA}$, and allowed more air to leak than the corresponding sizes of the LMA-U $(P<0.05)$. The size 3 of the Shiley LM performed similarly to the corresponding size of the LMA-U and maintained a better seal than the AA $(P<0.05)$.

\section{Shaft compressibility}

During use, the shaft of an LM may become compressed due to crimping or patient biting. The ability to compress the shaft of three sizes of the Shiley LM, LMA-U, and AA was assessed by measuring the change in the air pressure through the shaft before and after application of pressure to the shaft. The compression force applied ranged from 12 to $50 \mathrm{~N}$, depending on the device size (Table 1). The average bite force of adults depends on many factors including age, sex, and dentition, and tends to average $\sim 250-285 \mathrm{~N}$ at the first molar. ${ }^{6}$ The results of the compression test are shown in Table 5. The shaft of the Shiley LM was less compressible than those of the LMA-U and the AA for every size tested.

\section{Discussion}

Laryngeal masks cover and isolate the patient's tracheal inlet via an inflatable cuff. Generally, these devices consist of a dome surrounded by an inflatable cuff, a shaft, and an inflation system for inflation of the cuff. The Shiley LM introduces an inflation system integrated into the shaft, a difference from the LMA-U and the AA, in which the inflation tube is loose and the inflation system is bonded only at the cuff. The integrated inflation system of the Shiley laryngeal device is designed to simplify insertion by avoiding interference from a loose inflation tube. Additionally, integrating the inflation system into the shaft can prevent occlusion resulting from kinking or patient biting on the inflation tube.?

Laryngeal masks come in a range of sizes to fit patients of a wide range of body weights, from infants to large adults. The Shiley LM size offerings are identical to those of the LMA-U and the AA, including a size 6 for patients who weigh $>100 \mathrm{~kg}$. The dome and cuff of the sizes 1 and 3 Shiley LM were found to be less flexible than those of the corresponding sizes of the LMA-U and the AA, while the flexibility of the size 5 was intermediate to those of the AA and the LMA-U. The shaft of size 1 of the Shiley LM was also less flexible than those of the other two devices, and the size 3 shaft was less flexible than the shaft of the LMA-U but no different from the shaft of the AA. The flexibility of the size 5 shaft was intermediate to those of the size 5 of the other two devices. These differences in flexibility correlate to differences in stability. The connections between structures of the Shiley LM were much more resistant to failure than those of the LMA-U. This may prevent separation or tearing of the device under clinical scenarios in which stress is placed on the device. ${ }^{8}$

The conformation of the shaft of the Shiley LM differs from those of the LMA-U and the AA. First, the curvature of the shaft of the Shiley LM is identical to that of an endotracheal tube and therefore does not differ across different product sizes intended for different patients of different body weights. In contrast, the shafts of the other two devices vary with product size (Table 2). Second, the minimum internal diameter of the shaft of the Shiley LM is larger than that of the other devices for the four smallest product sizes (Table 2). The larger internal diameter could correlate to increased airflow and decreased work of breathing for small patients (body weight $<30 \mathrm{~kg}$ ). ${ }^{9}$ Third, the shaft of the Shiley LM is much more resistant to compression than those of the other two devices. This compression resistance could reduce the chance of crimping and also of occlusion due to patient biting, a common event that, in rare circumstances, may result in complications. ${ }^{7}$

Functionally, the different devices performed similarly in a test of sealing ability. The Shiley LM sealed as well as or better than either the LMA-U (size 3) or the AA (sizes 1 and 5).

\section{Study limitations}

The flat glass surface used in the sealing performance study does not model the actual environment of the trachea so may not accurately model the ability of the devices to form a seal in the clinical situation. Additionally, the tests were carried 
out at room temperature while the devices are used at body temperature, and flexibility and other characteristics may vary with temperature. The effects of differences in flexibility of the parts of the devices on ease of insertion or patient comfort were not examined.

\section{Conclusion}

The Shiley LM represents a new disposable laryngeal mask device. The results of the study demonstrate that the device resembles other commercially available laryngeal mask devices, though it exhibits greater tensile strength and lower flexibility. The unique integrated inflation tube present in the Shiley LM could make insertion easy and protect against occlusion of the inflation tube by patient biting on the tube. As this is a bench study, drawing clinical conclusions is limited as well. Further clinical work should be performed to evaluate the effectiveness of the Shiley LM in its new configuration relative to other commercially available laryngeal mask devices.

\section{Acknowledgment}

The authors would like to acknowledge Jason Cai for statistical assistance, and Tamara Golden for writing assistance.

\section{Disclosure}

YHZ and KJ work for Medtronic, the manufacturer of the Shiley ${ }^{\mathrm{TM}}$ laryngeal mask tested and discussed in this paper. The authors report no other conflicts of interest in this work.

\section{References}

1. van Zundert TC, Brimacombe JR, Ferson DZ, Bacon DR, Wilkinson DJ. Archie Brain: celebrating 30 years of development in laryngeal mask airways. Anaesthesia. 2012;67(12):1375-1385.

2. Ramachandran SK, Kumar AM. Supraglottic airway devices. Respir Care. 2014;59(6):920-931.

3. Verghese C, Berlet J, Kapila A, Pollard R. Clinical assessment of the single use laryngeal mask airway - the LMA-unique. Br JAnaesth. 1998;80(5): 677-679.

4. Asai T. Editorial II: who is at increased risk of pulmonary aspiration? Br J Anaesth. 2004;93(4):497-500.

5. Bosson N, Gordon P. Laryngeal mask airway. Medscape Drugs Dis. 2014. Available from: http://emedicine.medscape.com/article/82527 -overview - a1. Accessed August 25, 2014.

6. Takaki P, Vieira M, Bommarito S. Maximum bite force analysis in different age groups. Int Arch Otorhinolaryngol. 2014(18):272-276.

7. Devys JM, Balleau C, Jayr C, Bourgain JL. Biting the laryngeal mask: an unusual cause of negative pressure pulmonary edema. Can JAnaesth. 2000; 47(2):176-178.

8. Khoo ST. The laryngeal mask airway - an unusual complication. Anaesth Intensive Care. 1993;21(2):249-250.

9. Stankiewicz B, Darowski M, Glapinski J, Michnikowski M, Guć M, Kuraszkiewicz B. A new endotracheal tube for infants - laboratory and clinical assessment: a preliminary study. Paediatr Anaesth. 2013;23(5):440-445.
Medical Devices: Evidence and Research

\section{Publish your work in this journal}

Medical Devices: Evidence and Research is an international, peerreviewed, open access journal that focuses on the evidence, technology, research, and expert opinion supporting the use and application of medical devices in the diagnosis, monitoring, treatment and management of clinical conditions and physiological processes. The identification of novel

\section{Dovepress}

devices and optimal use of existing devices which will lead to improved clinical outcomes and more effective patient management and safety is a key feature. The manuscript management system is completely online and includes a quick and fair peer-review system. Visit http://www. dovepress.com/testimonials.php to read real quotes from authors. 